\title{
Erianin inhibits human cervical cancer cell through regulation of tumor protein p53 via the extracellular signal-regulated kinase signaling pathway
}

\author{
MENGTING LI ${ }^{1,2}$, YULIN HE ${ }^{1,2}$, CHENG PENG ${ }^{1,2}$, XIAOFANG XIE ${ }^{1,2}$ and GUANYING HU ${ }^{1,2}$ \\ ${ }^{1}$ State Key Laboratory Breeding Base of Systematic Research, Development and Utilization of Chinese Medicine Resources; \\ ${ }^{2}$ School of Pharmacy, Chengdu University of Traditional Chinese Medicine, Chengdu, Sichuan 611137, P.R. China
}

Received August 19, 2017; Accepted February 12, 2018

DOI: $10.3892 / \mathrm{ol} .2018 .9267$

\begin{abstract}
Erianin, a natural bibenzyl compound, is present in Dendrobium chrysotoxum Lindl. (commonly known as Shihu in China), which is used as an antipyretic and analgesic in traditional Chinese medicine, and has been reported to exert inhibitory effects on cancer cells in vitro. Cervical cancer is the third-most common cancer in women worldwide, and has the highest morbidity rate of gynecological malignancies. Thus, the identification of effective chemotherapeutical agents to treat this disease is urgent. The aim of the present study was to elucidate the biological functions and molecular mechanism of erianin on HeLa cells. Cellular proliferation was assessed using an MTT assay and flow cytometry assay with propidium iodide (PI) staining. Apoptosis rates were observed using a high content screening system via annexin V-fluorescein isothiocyanate/PI double staining, and measured by flow cytometry. The protein levels of tumor protein $\mathrm{p} 53$, extracellular signal-regulated kinase 1/2 (ERK1/2), caspase-3, B-cell lymphoma-2 (Bcl-2) and Bcl-2-associated X (Bax) were assessed by western blot analysis. Erianin inhibited the growth of HeLa cells and induced apoptosis in a dose- and time-dependent manner, inducing cell cycle arrest at the $G_{2} / M$ stage. Erianin treatment also increased the expression of Bax and caspase-3, but decreased levels of Bcl-2 and phosphorylated-ERK1/2. Cells treated with paclitaxel were regarded as the positive group. Together, the results of the present study indicated that erianin could be considered as an effective drug candidate; in HeLa cells it inhibited cellular proliferation and promoted apoptosis via regulation of the ERK1/2 signaling and mitochondrial-based apoptosis pathways. Thus, erianin
\end{abstract}

Correspondence to: Professor Cheng Peng or Dr Xiaofang Xie, School of Pharmacy, Chengdu University of Traditional Chinese Medicine, 1166 Liutai Road, Chengdu, Sichuan 611137, P.R. China E-mail: pengchengchengdu@126.com

E-mail: xxf14544@163.com

Key words: erianin, HeLa, proliferation, apoptosis, extracellular signal-regulated kinase pathway has the promise to be developed further for cervical cancer therapy.

\section{Introduction}

Cervical cancer, which is often induced by human papilloma virus (HPV) infection, is one of the most serious life-threatening diseases among women in developing countries $(1,2)$. Recently, two prophylactic vaccines, Cervarix and Gardasil, against the high-risk strains HPV-16 and HPV-18 have been developed and are used in more than 100 countries worldwide (3). However, these vaccines offer no benefit for patients that are already infected with HPV, have pre-cancerous lesions or cervical cancer (4). The long-term use of routine approaches to treat cervical cancer, including surgical removal, radiotherapy and chemotherapy, further damage the health of patients. Accordingly, seeking effective treatment agents from natural compounds to prevent and treat cervical cancer without these adverse effects is clinically urgent.

Erianin [2-Methoxy-5-(2-(3,4,5-trimethoxyphenyl)-ethyl)phenol] (Fig. 1) is a natural bibenzyl compound present in Dendrobium chrysotoxum Lindl., which is commonly known as Shihu in China; it has been used as a herbal drug for thousands of years in traditional Chinese medicine (TCM), where it is often used as antipyretic and analgesic medication (5). Previous studies have demonstrated that erianin can elicit multiple pharmacological effects, including anti-oxidative (6) and antitumor activity (7). Erianin has been reported to inhibit cell proliferation and induce apoptosis in human promyelocytic leukemia HL-60 cells (8), and reverse multidrug resistance in B16/hMDR-1 cells (9). However, the effect of erianin on cervical cancer HeLa cells and the underlying molecular mechanisms behind these effects remain unclear. Thus, in the present study, the anticancer effects of erianin in HeLa cells compared with paclitaxel, a frequently used chemotherapeutic drug, were examined. Additionally, the involvement of tumor protein p53 and extracellular signal-regulated kinase (ERK) signaling were also investigated as potential molecular mechanism. The present study aimed to elucidate the effects and potential mechanism of erianin on cervical cancer HeLa cells in vitro. 


\section{Materials and methods}

Reagents. Erianin and paclitaxel (PTX) were purchased from Chengdu Must Bio-Technology Co. Ltd. (Chengdu, China), purity $>98 \%$, and dissolved in dimethyl sulfoxide (DMSO) (Sigma-Aldrich; Merck KGaA, Darmstadt, Germany) for use.

Cell culture. HeLa cells, human cervical cancer cell line, were obtained from American Type Culture Collection (Manassas, VA, USA). HeLa cells were cultured in Dulbecco's modified Eagle's medium (DMEM) (Gibco; Thermo Fisher Scientific, Inc., Waltham, MA, USA) with $10 \%$ fetal bovine serum (FBS) (Gibco; Thermo Fisher Scientific, Inc.) at $37^{\circ} \mathrm{C}$ in a humidified atmosphere of $5 \% \mathrm{CO}_{2}$.

Cell viability assay. The cytotoxic activity of erianin on HeLa cells was assessed using an MTT assay. HeLa cells were treated with erianin at various concentrations $(3.9,7.8,15.7,31.4$ or $157.0 \mu \mathrm{M})$ for different time points $(24,48$ or $72 \mathrm{~h})$, and then incubated with MTT $(0.5 \mathrm{mg} / \mathrm{ml})$ at $37^{\circ} \mathrm{C}$ for $4 \mathrm{~h}$. Meanwhile, HeLa cells were treated with PTX (7.81, 15.63, 31.25, 62.50, 125.0 and $250.0 \mathrm{nM}$ ) for $48 \mathrm{~h}$ as the positive groups. The purple formazan crystals were dissolved in $0.15 \mathrm{ml}$ of DMSO. Following shaking, the plates read on an automated microplate spectrophotometer at $570 \mathrm{~nm}$. Assays were performed in triplicate in three independent experiments.

Cell cycle assay. Following exposure to erianin (3.9, 7.9, 15.7 and $31.4 \mu \mathrm{M}$ ) and $0.05 \mu \mathrm{M}$ PTX for $12,24,36$ and $48 \mathrm{~h}$, the control (without treatment) and experimental groups $\left(3.0 \times 10^{5}\right.$ cells in a $60-\mathrm{mm}$ dish) were harvested using $0.25 \%$ trypsin, washed with ice-cold PBS and collected by centrifugation at $400 \times \mathrm{g}$ for $15 \mathrm{~min}$ at $4^{\circ} \mathrm{C}$. The cells were suspended in PBS and fixed with $70 \%$ ethanol (v/v) overnight at $4^{\circ} \mathrm{C}$. The following day, subsequent to three washes with ice-cold PBS, the cells were treated with $100 \mu 1 \mathrm{RNase}$ at $37^{\circ} \mathrm{C}$ for $30 \mathrm{~min}$ and stained with $400 \mu 1$ propidium iodide (PI) (Nanjing KeyGen Biotech Co. Ltd., Nanjing, China) for $30 \mathrm{~min}$. Next, cell cycle analysis was performed by flow cytometry (Coulter Epics XL; Beckman Coulter, Inc., Brea, CA, USA). The data were analyzed using MultiCycle software (version 6-16-03-F32; Beckman Coulter, Inc.).

Cell apoptosis assay. For apoptotic cells analysis, HeLa cells were treated with various concentrations of erianin $(3.9,7.9$, 15.7, 31.4) $\mu \mathrm{M}$ and $0.05 \mu \mathrm{M}$ PTX for $12,24,36$ and $48 \mathrm{~h}$. The cells were then collected and washed with ice-cold PBS. Next, early apoptosis was measured using an Annexin V-Fluorescein Isothiocyanate (FITC)/PI apoptosis detection kit (BD Pharmingen; BD Biosciences, Franklin Lakes, NJ, USA). The cells were suspended with $100 \mu \mathrm{l}$ of binding buffer and stained with $5 \mu \mathrm{l}$ annexin V-FITC and $5 \mu \mathrm{l}$ PI for $20 \mathrm{~min}$ at room temperature in the dark and then $400 \mu \mathrm{l}$ binding buffer was added. The proportion of apoptotic cells were determined by flow cytometry and observed using the ImageXpress ${ }^{\circledR}$ Micro XLS High Content Screening system (Molecular Devices, LLC, Sunnyvale, CA, USA), with Hoechest 33342 staining.

Western blot assay. Cells were treated with 3.9, 7.8, 15.7 or $31.4 \mu \mathrm{M}$ erianin or $0.05 \mu \mathrm{M}$ PTX for $48 \mathrm{~h}$, lysed using lysis

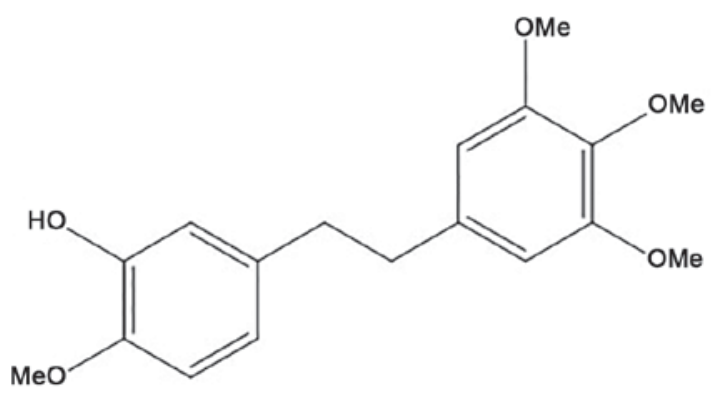

Figure 1. Chemical structure of erianin. $\mathrm{C}_{18} \mathrm{H}_{22} \mathrm{O}_{5}$; molecular weight, 318.36.

buffer radioimmunoprecipitation assay (RIPA; $3 \mathrm{ml}$; cat. no. AR0102; Wuhan Boster Biological Technology, Ltd., Wuhan, China) buffer with phenylmethane sulfonyl fluoride (40 $\mu \mathrm{l}$; cat. no. AR1778; Wuhan Boster Biological Technology, Ltd.) and a protease inhibitor cocktail (cat. no. P8340; $30 \mu \mathrm{l}$ Sigma-Aldrich; Merck KGaA) and the lysates were measured for protein concentrations with a total protein quantitative assay (Coomassie brilliant blue method) kit (cat. no. A405-2; Nanjing Jiancheng Bioengineering Institute, Nanjing, China) as described previously (10), according to the manufacturer's protocols. Protein samples $(20 \mu \mathrm{g})$ were separated by $10 \%$ SDS-PAGE and then transferred to a polyvinylidene fluoride membrane. The membrane was blocked for $2 \mathrm{~h}$ with $5 \%$ non-fat dry skimmed milk. Following incubation overnight at $4^{\circ} \mathrm{C}$ with antibodies against tumor protein p53 (hereafter p53) (dilution, 1:1,000; cat. no. ab131442; Abcam, Cambridge, UK), ERK1/2 (dilution, 1:1,000; cat. no. ab50011; Abcam), phosphorylated ERK1/2 (dilution, 1:1,000; cat. no. ab36991; Abcam), caspase-3 (dilution, 1:1,000; cat. no. 9665; Cell Signaling Technology, Inc., Danvers, MA, USA), B-cell lymphoma-2 (Bcl-2) (dilution, 1:1,000; cat. no. ab136285; Abcam), Bcl-2-associated X (Bax) (dilution, 1:1,000; cat. no. ab32503; Abcam), GAPDH (dilution, 1:1,000; cat. no. BA2913; Wuhan Boster Biological Technology, Ltd.) and $\beta$-actin (dilution, 1:1,000; cat. no. ab3280, Abcam), the membrane was incubated with horseradish peroxidase-conjugated anti-rabbit antibody (dilution, 1:5,000; cat. no. RA1054; Wuhan Boster Biological Technology, Ltd.) at $37^{\circ} \mathrm{C}$ for $2 \mathrm{~h}$. Finally, the reaction was visualized using SuperLumia enhanced chimiluminescence kit (cat. no. K22020; Abbkine Scientific Co., Ltd, Wuhan, China) and detected using a Molecular Imager ${ }^{\circledR}$ Gel Doc ${ }^{\text {TM }}$ $\mathrm{XR}^{+}$(Bio-Rad Laboratories, Inc., Hercules, CA, USA). The integrated density of the bands was quantified using Image Lab 5.1 software (Bio-Rad Laboratories, Inc.).

Bioinformatics methods. The data concerning the association between p53 expression and survival ratios in patients with cervical cancer were acquired from The Cancer Genome Atlas (TCGA) database (https://cancergenome.nih.gov/). The interaction regulatory function of p53, ERK1/2, Bax and Bcl-2 was analyzed using Pathway Commons (http://www.pathwaycommons.org/) (11).

Statistical analysis. All experiments were independently repeated three times. All quantitative data were presented as the mean \pm standard deviation and analyzed using SPSS 19.0 

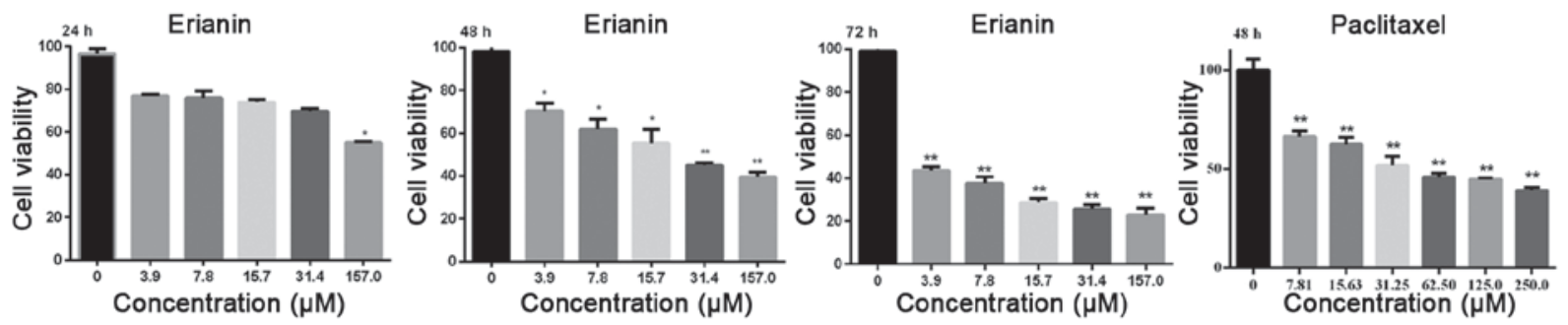

Figure 2. Changes of proliferation ability of HeLa cells following treatment with erianin and paclitaxel. The viability of HeLa cells at different time points were evaluated by MTT following incubated with different concentrations of erianin. ${ }^{*} \mathrm{P}<0.05,{ }^{* * *} \mathrm{P}<0.01$.

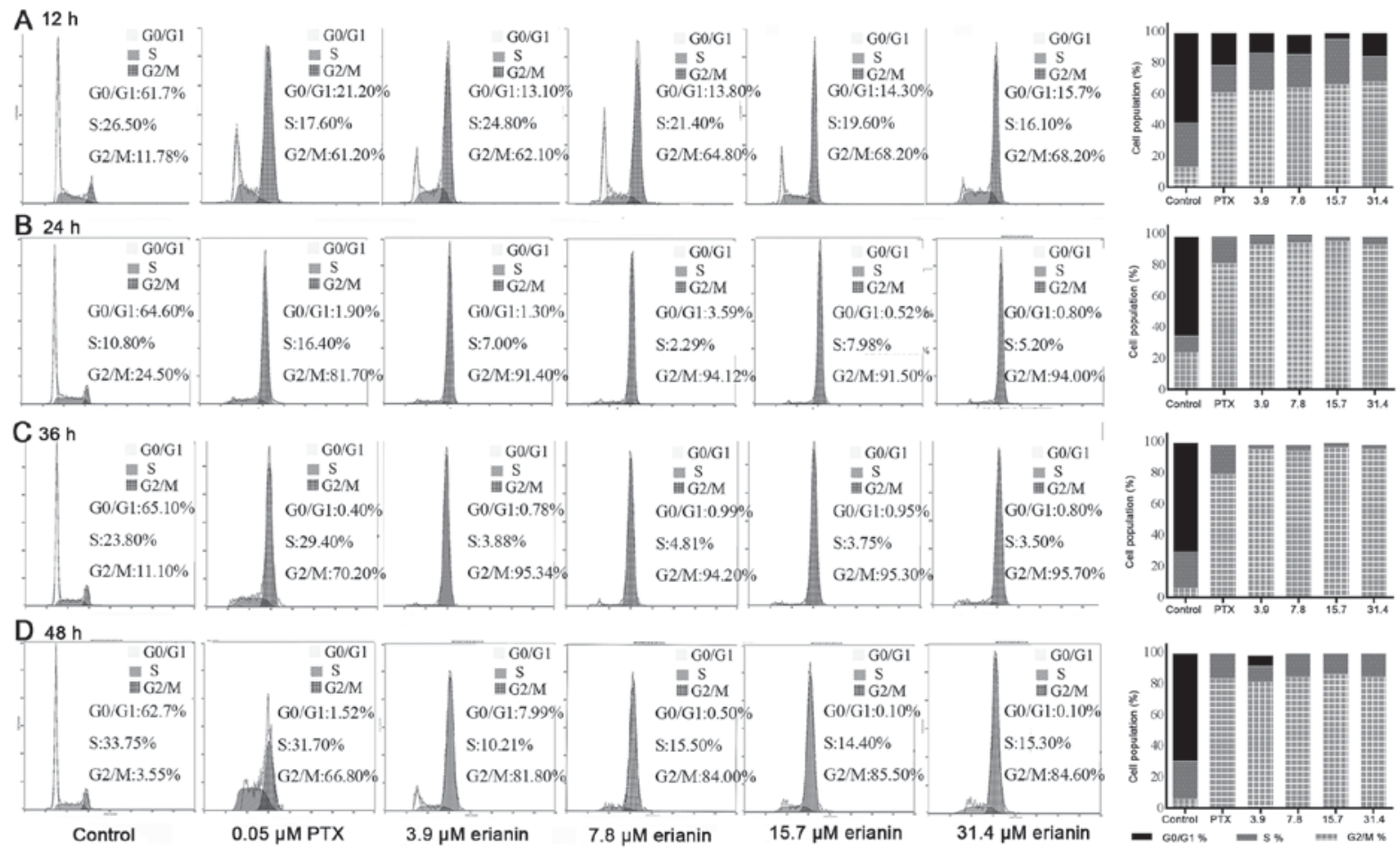

Figure 3. Effects of erianin on cell cycle of HeLa. (A) HeLa cells were harvested following treatment with the indicated concentration of erianin or PTX for (A) 12, (B) 24, (C) 36 and (D) $48 \mathrm{~h}$. Data are presented as the mean. PTX, paclitaxel.

software (IBM Corp., Armonk, NY, USA). The half-maximal inhibitory concentration $\left(\mathrm{IC}_{50}\right)$ and combination index values were calculated using a logit regression model. One-way analysis of variance with a least significant difference post-hoc test was used to compare mean values between the control and treatment groups. $\mathrm{P}<0.05$ was considered to indicate a statistically significant difference.

\section{Results}

Effects of erianin on the proliferation of HeLa cells. To evaluate the inhibitory effect of erianin on HeLa cells, cell viability was assessed using an MTT assay after 24,48 , or $72 \mathrm{~h}$ of treatment. The results showed that erianin inhibited HeLa cells growth in a dose- and time-dependent manner. At doses of 3.9, 7.8, 15.7, 31.4 and $157.0 \mu \mathrm{M}$, erianin elicited a significant inhibition in HeLa cell viability after a 48 -h treatment $(\mathrm{P}<0.05)$. The $\mathrm{IC}_{50}$ values of erianin and PTX after $48 \mathrm{~h}$ were $8.3 \pm 1.3$ and $0.055 \pm 0.0017 \mu \mathrm{M}$, respectively (Fig. 2).
Effects of erianin on cell cycle of HeLa cells. To investigate whether the inhibition in cell proliferation induced by erianin could be attributed to the induction of cell cycle arrest, cell cycle analysis was performed. This analysis revealed that erianin induced cell cycle arrest in a time- and dose-dependent manner (Fig. 3). Compared with the control, the proportion of cells in $\mathrm{G}_{0} / \mathrm{G}_{1}$ phase following treatment with erianin and PTX decreased significantly after $12 \mathrm{~h}$ of treatment $(\mathrm{P}<0.01)$, whereas the proportion of cells in $\mathrm{G}_{2} / \mathrm{M}$ phase increased significantly $(\mathrm{P}<0.01)$ (Fig. 3A), indicating that erianin could at least partly inhibit HeLa cells proliferation via $\mathrm{G}_{2} / \mathrm{M}$ checkpoint arrest.

Effects of erianin on apoptosis of HeLa cells. Induction of apoptosis is an central mechanism by which anticancer drugs can inhibit cancer cell growth. Flow cytometry analysis demonstrated that erianin at a dose of $7.8 \mu \mathrm{M}$ induced apoptosis rate compared with the control, $0.22 \pm 0.26$ vs. $1.52 \pm 0.29 \%$ at $12 \mathrm{~h}$, $0.10 \pm 0.1$ vs. $8.12 \pm 0.92 \%$ at $24 \mathrm{~h}, 1.83 \pm 0.71$ vs. $11.53 \pm 1.22 \%$ at 36 $\mathrm{h}$ and $5.99 \pm 5.12$ vs. $41.83 \pm 5.32 \%$ at 48 h, respectively (Fig. 4). 

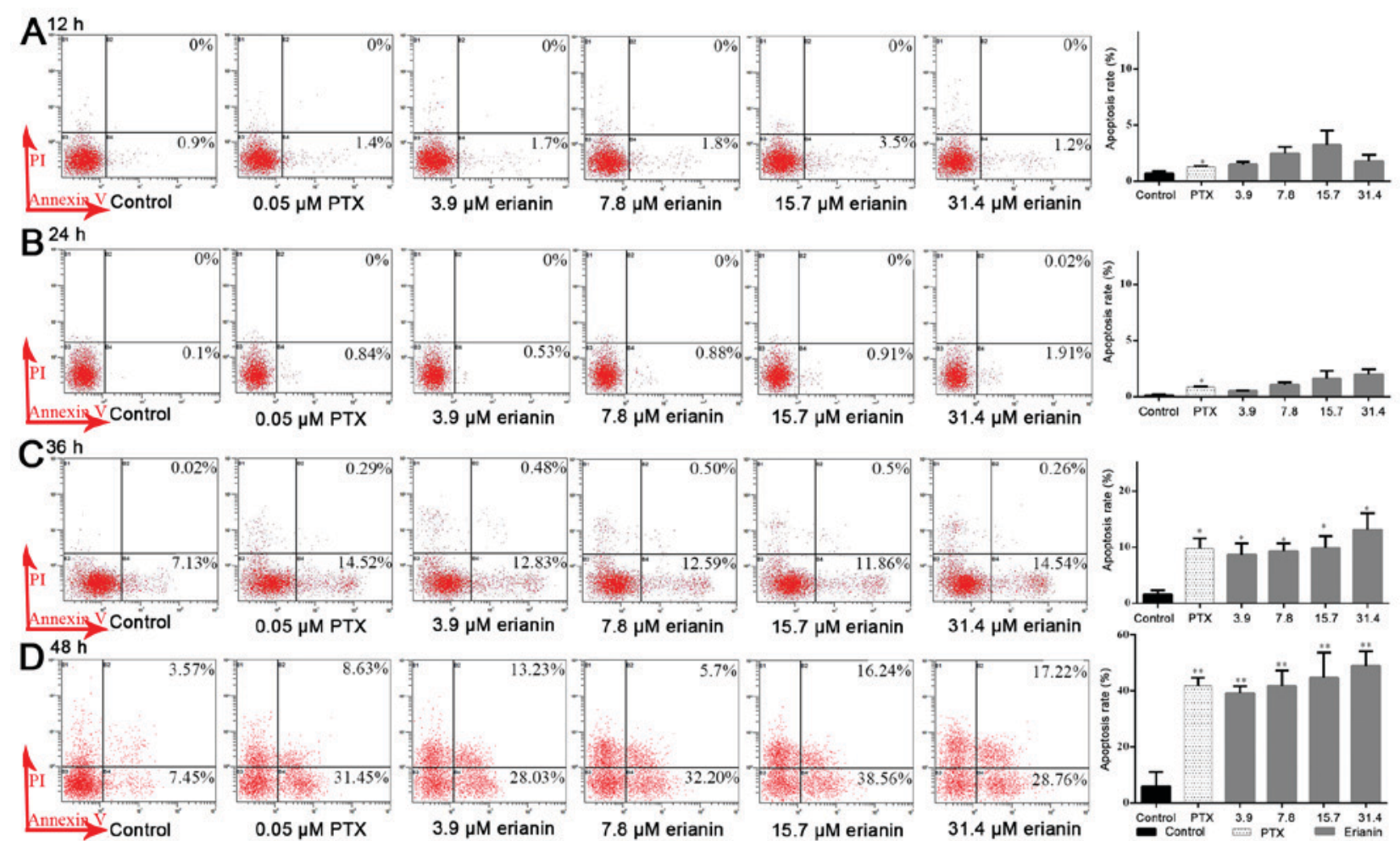

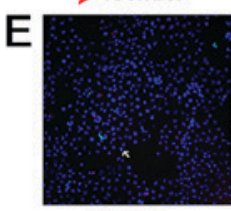

Control

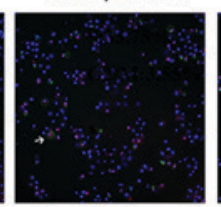

$0.05 \mu \mathrm{M}$ PTX

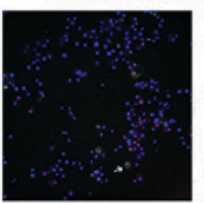

$3.9 \mu \mathrm{M}$ erianin

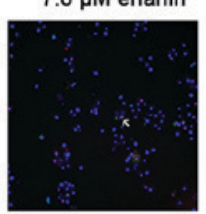

$7.8 \mu \mathrm{M}$ erianin

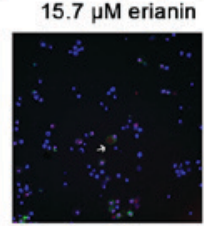

$15.7 \mu \mathrm{M}$ erianin

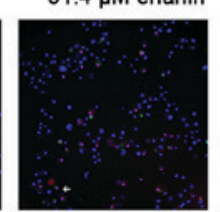

$31.4 \mu \mathrm{M}$ erianin

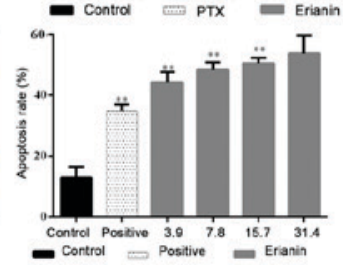

Figure 4. Effects of erianin on apoptosis of HeLa. (A) HeLa cells were harvested and stained following erianin or PTX treatment for 12, (B) 24, (C) 36 and (D) 48 h. (E) Images were acquired when HeLa were exposed to erianin for $48 \mathrm{~h}$, and early apoptotic cells were marked. ${ }^{*} \mathrm{P}<0.05$, ${ }^{* *} \mathrm{P}<0.01$. PTX, paclitaxel; PI, propidium iodide.

As shown in Fig. 4D, erianin could increase rates of early apoptosis in HeLa cells, from $5.99 \pm 5.12 \%$ in the control (untreated cells at $48 \mathrm{~h}$ ) to $48.91 \pm 5.22 \%$ in cells treated with $31.4 \mu \mathrm{M}$ erianin after $48 \mathrm{~h}$ of treatment $(\mathrm{P}<0.05)$. Therefore, the results of the present study demonstrated that erianin induced apoptosis in HeLa cells in a time- and dose-dependent manner.

Effects of erianin on the expression of p53, ERK1/2, caspase-3, $B c l-2$ and Bax proteins. It has been reported that the p53 and ERK pathways are involved in mitochondrial-based apoptosis $(12,13)$. Therefore, whether erianin induced mitochondrial-based apoptosis through the inhibition of ERK1/2 signaling and activation of p53 was examined. The results of western blot analysis revealed that erianin could reduce the levels of p53 expression and phosphorylated-ERK1/2 expression without decreasing total ERK1/2 expression, indicating that erianin could affect ERK1/2 phosphorylation in a concentration-dependent manner, compared with the control $(\mathrm{P}<0.05$; Fig. 5A and B). Further study indicated that administration of erianin for $48 \mathrm{~h}$ evidently promoted caspase-3 cleavage, upregulated the expression of $\mathrm{Bax}$ and downregulated the expression of Bcl-2 ( $\mathrm{P}<0.05$; Fig. 5C and D), Note that, since the effect of erianin on $\mathrm{p}$-ERK1/2 and caspase-3 expression at a concentration of $1.9 \mu \mathrm{M}$ was similar to that at $3.9 \mu \mathrm{M}$, these data were therefore not included in the figure. A concentration of $3.9 \mu \mathrm{M}$ as the minimum concentration was selected for the further investigation.

ERK and p53 expression are associated with mitochondrial-based apoptosis in cervical cancer. p53 is an essential tumor suppressor gene in various types of cancer(14). According to data from TGCA, high expression of p53 was positively associated with the survival rates in patients with cervical cancer $(\mathrm{P}=0.038)$ (https://cancergenome.nih.gov/), indicating that promoting p53 expression may represent a promising approach to treating cervical cancer (Fig. 6A). Furthermore, data from Pathway Commons (http://www.pathwaycommons. org/) in cervical cancer revealed that p53 serves essential roles in numerous different pathways, including apoptosis. Specially, p53 could control the expression and activation of Bcl-2 and Bax, and control phosphorylation of ERK1/2. The activation of the ERK1/2 pathway could control the activity of Bcl-2 (Fig. 6B).

\section{Discussion}

An epidemiological study indicated that in 2008, cervical cancer was the third-most common cancer in women worldwide and the gynecological malignancy with the highest morbidity, with an estimated 529,000 new cases 

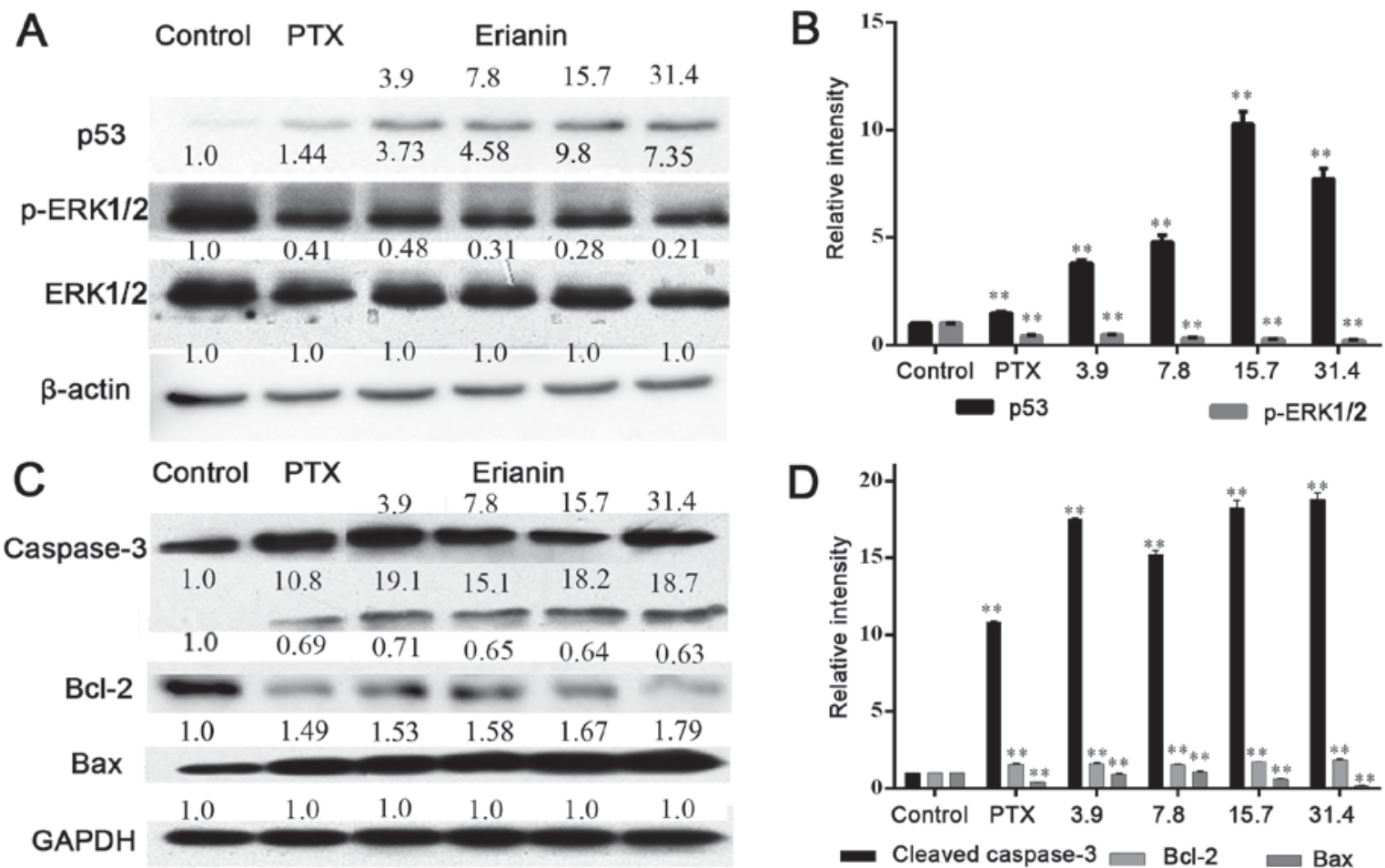

Figure 5. Effect of erianin on p53/ERK1/2 signaling. (A) The levels of p53, ERK1/2 and its phosphorylated protein were tested after treatment for $48 \mathrm{~h}$ by $0.05 \mu \mathrm{M}$ PTX and erianin at concentrations of 3.9, 7.8, 15.7 and $31.4 \mu \mathrm{M}$ for HeLa cells, with (B) quantification of this expression. (C) The levels of caspase-3, Bcl-2 and Bax proteins were measured following treatment with compounds, with (D) quantification of this expression. ${ }^{* *} \mathrm{P}<0.01$. p53, tumor protein p53; p-ERK1/2, phosphorylated extracellular signal-regulated kinase 1/2; PTX, paclitaxel; Bcl-2, B-cell lymphoma-2; Bax, Bcl-2-assocated X; PTX, paclitaxel.
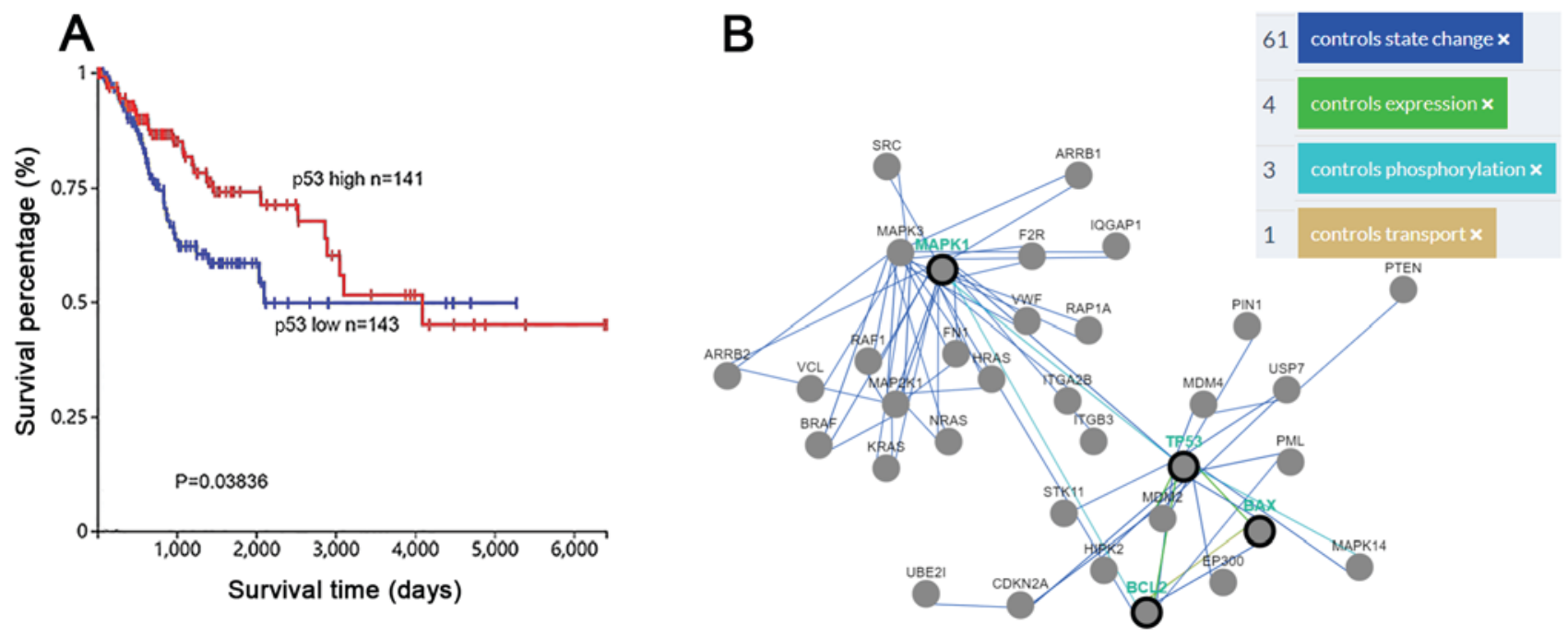

Figure 6. Role of $\mathrm{p} 53$ and ERK in mitochondrial-based apoptosis of cervical cancer. (A) Association between p53 expression and survival ratio. (B) Association between p53, ERK, Bax, and Bcl-2. p53, tumor protein p53; ERK, extracellular signal-regulated kinase; Bcl-2, B-cell lymphoma-2; Bax, Bcl-2-associated X.

occurring, resulting in 274,000 mortalities (15). Treatments for cervical cancer, including surgery, chemotherapy, radiation therapy, and chemotherapy combined with radiation therapy, can have a curative effect; however, these treatments are accompanied by adverse effects and recurrence within a short time (16-20). Accordingly, seeking effective chemotherapeutical agents to increase the curative rate, reduce the risk of recurrence and metastasis, and improve patient quality of life is highly desirable.
The development of antitumor drugs, particularly those based on natural products, is receiving considerable attention. Erianin, a naturally occurring product isolated from Dendrobium chrysotoxum Lindl., was reported to exert antitumor (21-23) and anti-oxidative effects (8). However, the anti-cervical cancer activity and the potential mechanisms of erianin have, to the best of our knowledge, not been assessed. The present study aimed to elucidate the effects of erianin on cell growth and apoptosis, and clarify the potential mechanism 
by which this occurred, which was associated with regulation of the ERK1/2 signaling and mitochondrial pathways.

Investigating the molecular mechanisms of cancer cell growth is critically important. Cell cycle dysregulation is a major factor in cancer cell growth (24). ERKs have a key role in promoting cell survival and cellular proliferation $(25,26)$ and have been considered to be significant targets for cancer therapeutics (27). Evidence indicates that erianin affects cell cycle progression, evidenced by inhibition of gastric carcinoma SGC-7901 cell proliferation by blocking progression to $S$ phase (28), and arresting progression at the $G_{2} / M$ phase in hepatocellular carcinoma Huh7 cells (23) and human colorectal cancer SW480 cells (29). In the present study, erianin exhibited potent anti-proliferative activities against HeLa cells ( $\mathrm{IC}_{50}=8.3 \pm 1.3 \mu \mathrm{M}$; Fig. 2$)$ at $48 \mathrm{~h}$, induced the arrest of HeLa cells at the $\mathrm{G}_{2} / \mathrm{M}$ phase (Fig. 3), and suppressing the phosphorylation of ERK1/2.

As a tumor suppressor, p53 has a pivotal role in cell cycle progression, the DNA damage response and apoptosis (12). Previous studies confirmed that p53 was one of the most commonly mutation genes in cancer (30-32). Additionally, the upregulation of p53 in cancer cells may prevent cancer cell proliferation by promoting cell cycle arrest and apoptosis (33-36). Liu et al (37) revealed that the p53 pathway in human cervical cancer cells is activated by reversion-inducing-cysteine-rich protein with Kazal motifs overexpression, which induces cancer cell apoptosis and reduces migration. In the present study, erianin treatment of HeLa cells could promote the activation of p53.

Apoptosis maintains the healthy survival/death balance in metazoan cells. Defects in apoptosis can cause cancer autoimmunity, whereas enhanced apoptosis may cause degenerative diseases (38). Mitochondria have a notable role in the intrinsic pathway of mammalian apoptosis. In various types of cancer, inhibitors of apoptosis are highly expressed whereas apoptosis promoters are mostly inactivated, resulting in a certain degree of drug resistance. Thus, reactivation of the normal apoptosis response in cancer cells through regulating apoptotic regulators is a desirable treatment approach $(39,40)$. Developing therapeutic approaches has involved modifying the activity of Bcl-2 family proteins to reactivate apoptosis, eradicating cancer cells (41-43). It is generally recognized that Bcl-2, regarded as an anti-apoptotic protein, was suppressed and pro-apoptotic proteins like Bax was overexpressed following cleavage of caspase-family proteins once cytochrome $\mathrm{C}$ was released from the mitochondria into the cytoplasm $(44,45)$. Evidence indicates provided that baicalein and betulinic acid induced apoptosis in HeLa cells by downregulating Bcl-2 expression and upregulating that of expression and caspase-8. When HeLa cells were exposed to erianin, the apoptotic rate was increased and expression of Bax was upregulated, caspase- 3 was activated and Bcl-2 levels reduced, which was consistent with the results of previous studies $(46,47)$, indicating that apoptosis induced by erianin was associated with the activation of the proteolytic caspase family and the $\mathrm{Bcl}-2$ family.

Paclitaxel exerts an anticancer function and has been manufactured into albumin-bound paclitaxel (nab-paclitaxel), which received US Food and Drug Administration approval for the treatment of metastatic breast cancer, non-small cell lung cancer and pancreatic cancer (48). The National Comprehensive Cancer Network recommends paclitaxel combined with carboplatin or cisplatin for treatment of recurrent or metastatic cervical cancer. Therefore, in the current study paclitaxel was used as positive control agent and was compared with erianin in terms of effects on HeLa cell proliferation, cell cycle and apoptosis. The results of this analysis revealed that erianin arrested progression at the $G_{2} / M$ phase and enhanced the apoptotic rate, similar to paclitaxel, indicating that erianin has the potential to serve as an anti-cervical cancer drug.

In conclusion, erianin is a promising anticancer compound, owing to its ability to inhibit growth of HeLa by arresting progression at the $\mathrm{G}_{2} / \mathrm{M}$ phase transition and inducing apoptosis. The potential mechanism of action involves regulation of the ERK1/2 signaling and mitochondrial pathways.

\section{Acknowledgements}

Not applicable.

\section{Funding}

The present study was financially supported by the National Natural Science Foundation of China (grant no. J1310034).

\section{Availability of data and materials}

All data generated or analyzed during the present study are included in this published article.

\section{Authors' contributions}

ML performed the cell cycle and apoptosis experiments. YH performed the cell viability experiments. GH participated in statistical analyses and wrote the manuscript. XX and $\mathrm{CP}$ designed the research and wrote the manuscript. All authors read and approved the final manuscript.

\section{Ethics approval and consent to publish}

Not applicable.

\section{Patient consent for publication}

Not applicable.

\section{Competing interests}

The authors declare that they have no competing interests.

\section{References}

1. Tsikouras P, Zervoudis S, Manav B, Tomara E, Iatrakis G, Romanidis C, Bothou A and Galazios G: Cervical cancer: Screening, diagnosis and staging. J BUON 21: 320-325, 2016

2. Bonneau C, Perrin M, Koskas M, Genin AS and Rouzier R: Epidemiology and risk factors for cancer of the uterus. Rev Prat 64: 774-779, 2014.(In French).

3. Castle PE and Maza M: Prophylactic HPV vaccination: Past, present, and future. Epidemiol Infect 144: 449-468, 2016.

4. Tang J and Hao F: The present situation and the future of vaccines for cervical cancer. Immunol J 26: 546-550, 2010. 
5. Chinese Pharmacopoeia Committee: Pharmacopoeia of China. Chin Med Sci Technol Press: 92-93, 2015.

6. Ng TB, Liu F and Wang ZT: Antioxidative activity of natural products from plants. Life Sci 66: 709-723, 2000.

7. Ma GX, Xu GJ and Xu LS: Inhibitory effects of Dendrobium chrysotoxum and its constituents on the mouse HePA and ESC. J Chin Pharm Univ 25: 188-189, 1994.

8. Li YM, Wang HR and Liu GQ: Erianin induces apoptosis in human leukemia HL-60 cells. Acta Pharmacol Sin 22: 1018-1022, 2001.

9. Ma GX and LeBlanc GA: The activity of erianin and chrysotoxine from Dendrobium chrysotoxum to reverse multidrug resistance in B16/hMDR-1 Cells. J Chin Pharm Sci 7: 142-146, 1998.

10. Zhang W, Yuan W, Xu N, Li J and Chang W: Icariin improves acute kidney injury and proteinuria in a rat model of pregnancy-induced hypertension. Mol Med Rep 16: 7398, 2017.

11. Cerami EG, Gross BE, Demir E, Rodchenkov I, Babur O, Anwar N, Schultz N, Bader GD and Sander C: Pathway Commons, a web resource for biological pathway data. Nucleic Acids Res 39: D685-D690, 2011.

12. Wawryk-Gawda E, Chylińska-Wrzos P, Lis-Sochocka M, Chłapek K, Bulak K, Jędrych M and Jodłowska-Jędrych B: P53 protein in proliferation, repair and apoptosis of cells Protoplasma 251: 525-533, 2014

13. Yang T, Xu F, Sheng Y, Zhang W and Chen Y: A targeted proteomics approach to the quantitative analysis of ERK/ Bcl-2-mediated anti-apoptosis and multi-drug resistance in breast cancer. Anal Bioanal Chem 408: 7491-7503, 2016.

14. Robles AI and Harris CC: p53-mediated apoptosis and genomic instability diseases. Acta Oncol 40: 696-701, 2001.

15. Ferlay J, Shin HR, Bray F, Forman D, Mathers C and Parkin DM: Estimates of worldwide burden of cancer in 2008: GLOBOCAN 2008. Int J Cancer 127: 2893-2917, 2010.

16. Long HJ 3rd, Bundy BN, Grendys EC Jr, Benda JA, McMeekin DS, Sorosky J, Miller DS, Eaton LA and Fiorica JV; Gynecologic Oncology Group Study: Randomized phase III tria of cisplatin with or without topotecan in carcinoma of the uterine cervix: A gynecologic oncology group study. J Clin Oncol 23: 4626-4633, 2005.

17. Kurtz JE, Freyer G, Joly F, Gladieff L, Kaminski MC, Fabbro M, Floquet A, Hardy-Bessard AC, Raban N, Ray-Coquard I and Pujade-Lauraine E; GINECO Group, France: Combined oral topotecan plus carboplatin in relapsed or advanced cervical cancer: A GINECO phase I-II trial. Anticancer Res 32: $1045-1049,2012$

18. Xiong Y, Liang LZ, Cao LP, Min Z and Liu JH: Clinical effects of irinotecan hydrochloride in combination with cisplatin as neoadjuvant chemotherapy in locally advanced cervical cancer. Gynecol Oncol 123: 99-104, 2011.

19. Peng R and Zhao CQ: Chemotherapy drugs in the application and research progress of cerical cancer treatment. Chin Pharm 24: 1143-1146, 2013

20. Jin ZH, Liao GH and Jiang N: Clinical analyses on 91 recurrence or metastasis cases of young women cervical cancer. Pract J Cancer 21: 502-503,511, 2006.

21. Sun J, Fu X, Wang Y, Liu Y, Zhang Y, Hao T and Hu X: Erianin inhibits the proliferation of T47D cells by inhibiting cell cycles, inducing apoptosis and suppressing migration. Am J Trans Res 8: 3077-3086, 2016

22. Yu Z, Zhang T, Gong C, Sheng Y, Lu B, Zhou L, Ji L and Wang Z: Erianin inhibits high glucose-induced retinal angiogenesis via blocking ERK1/2-regulated HIF-1 $\alpha$-VEGF/VEGFR2 signaling pathway. Sci Rep 6: 34306, 2016

23. Su P, Wang J, An JX, Zhu Q, Lu X and Tang Y: Inhibitory effect of erianin on hepatocellular carcinoma (HCC) Huh7 cells. Chin J Appl Environ Biol 17: 662-665, 2011.

24. Hanahan D, Weinberg RA. Hallmarks of cancer: the next generation. Cell. 2011; 144: 646-674.

25. El-Baba C, Mahadevan V, Fahlbusch FB, Mohan SS, Rau TT, Gali-Muhtasib $\mathrm{H}$ and Schneider-Stock R: Thymoquinone-induced conformational changes of PAK1 interrupt prosurvival MEK-ERK signaling in colorectal cancer. Mol Cancer 13: 201, 2014.

26. Song X, Wang Y, Du H, Fan Y, Yang X, Wang X, Wu X and Luo C: Overexpression of HepaCAM inhibits cell viability and motility through suppressing nucleus translocation of androgen receptor and ERK signaling in prostate cancer. Prostate 74 $1023-1033,2014$
27. Bai LX, Mao R, Wang J, Ding L, Jiang S, Gao C, Kang H, Chen X, Sun $\mathrm{X}$ and $\mathrm{Xu}$ J: ERK1/2 promoted proliferation and inhibited apoptosis of human cervical cancer cells and regulated the expression of c-Fos and c-Jun proteins. Med Oncol 32: 57, 2015.

28. Wei H, Shenglin M, Lingbin D, et al: Experimental study of erianin inducing apotosis in gastric carcinoma SGC-7901. Chin Cancer 17: 499-501, 2008 (In Chinese).

29. Cui XQ, Su P, Zhu QY, An JX, Wang J, Wu J and Tang YX: Molecular mechanism of apoptosis of human colorectal cancer SW480 cells induced by Erianin. Chin J Appl Environ Biol 17: 512-516, 2011.

30. Hoogervorst EM, Van SH and De VA: Nucleotide excision repair- and p53-deficient mouse models in cancer research. Mutat Res 574:3-21, 2015.

31. Haga S, Nakayama M, Tatsumi K, Maeda M, Imai S, Umesako S, Yamamoto H, Hilgers J and Sarkar NH: Overexpression of the p53 gene product in canine mammary tumors. Oncol Rep 8: 1215-1219, 2001

32. Park YK, Chi SG, Kim YW, Park HR and Unni KK: P53 mutations in Ewing's sarcoma. Oncol Rep 8: 533-537, 2001.

33. Moskovits N, Kalinkovich A, Bar J, Lapidot T and Oren M: p53 attenuates cancer cell migration and invasion through repression of SDF-1/CXCL12 expression in stromal fibroblasts. Cancer Res 66: 10671-10676, 2006

34. Khan S, Chib R, Shah BA, Wani ZA, Dhar N, Mondhe DM, Lattoo S, Jain SK, Taneja SC and Singh J: A cyano analogue of boswellic acid induces crosstalk between p53/PUMA/Bax and telomerase that stages the human papillomavirus type 18 positive HeLa cells to apoptotic death. Eur J Pharmacol 660: 241-248, 2011.

35. Chen ZL, Gu PQ, Liu K, Su YJ and Gao LJ: The globular heads of the $\mathrm{Clq}$ receptor regulate apoptosis in human cervical squamous carcinoma cells via a p53-dependent pathway. J Transl Med 10: $1-12,2012$.

36. Zhou MJ, Chen FZ, Chen HC, Wan XX, Zhou X, Fang Q and Zhang DZ: ISG15 inhibits cancer cell growth and promotes apoptosis. Int J Mol Med 39: 446-452, 2017.

37. Liu Y, Li L, Liu Y, Geng P, Li G, Yang Y and Song H: RECK inhibits cervical cancer cell migration and invasion by promoting p53 signaling pathway. J Cell Biochem 4: 3058-3066, 2018

38. Hassan M, Watari H, AbuAlmaaty A, Ohba Y and Sakuragi N: Apoptosis and molecular targeting therapy in cancer. Biomed Res Int 2014: 150845, 2014

39. Kaufmann SH and Vaux DL: Alterations in the apoptotic machinery and their potential role in anticancer drug resistance. Oncogene 22: 7414-7430, 2003.

40. Schmitt CA, Rosenthal CT and Lowe SW: Genetic analysis of chemoresistance in primary murine lymphomas. Nat Med 6:1029-1035, 2000

41. Kang MH and Reynolds CP: Bcl-2 inhibitors: Targeting mitochondrial apoptotic pathways in cancer therapy. Clin Cancer Res 15:1126-1132, 2009

42. Patel MR, Masood A, Patel PS and Chanan-Khan AA: Targeting the Bcl-2. Curr Opin Oncol 21: 516-523, 2009.

43. Leibowitz B and Yu J: Mitochondrial signaling in cell death via the Bcl-2 family. Cancer Biol Ther 9: 417-422, 2010.

44. Hu Y, Ding L, Spencer DM and Núñez G: WD-40 repeat region regulates Apaf-1 self-association and procaspase-9 activation. J Biol Chem 273: 33489-33494, 1998.

45. Jiang XJ and Wang XD: Cytochrome C-mediated apoptosis. AnnuRev Biochem 73: 87-106, 2014

46. Peng Y, Guo C, Yang Y, Li F, Zhang Y, Jiang B and Li Q: Baicalein induces apoptosis of human cervical cancer HeLa cells in vitro. Mol Med Rep 11: 2129-2134, 2015.

47. Xu T, Pang Q, Zhou D, Zhang A, Luo S, Wang Y and Yan X: Proteomic investigation into betulinic acid-induced apoptosis of human cervical cancer HeLa cells. PLoS One 9: e105768, 2014.

48. Kundranda MN and Niu J: Albumin-bound paclitaxel in solid tumors: Clinical development and future directions. Drug Des Devel Ther 9: 3767-3777, 2015

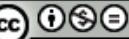

This work is licensed under a Creative Commons Attribution-NonCommercial-NoDerivatives 4.0 International (CC BY-NC-ND 4.0) License. 